Assiut Scientific Nursing Journal

http://asnj.journals.ekb.eg

http://www.arabimpactfactor.com

DOI: $10.21608 / A S N J .2021 .85050 .1212$

\title{
Illness Perception among Psychiatric Patients and its Impact on Internalized Stigma and Medication Adherence
}

\author{
Sonia M. El-Sayad
}

Assistant Professor Psychiatric Nursing and Mental Health, Faculty of Nursing- Port-Said University, Egypt.

\begin{abstract}
Background: The extent to which patients perceive and cognitively represent their illness, affords healthcare providers references concerning how they can manage patients' disorders and how will be the outcomes. Aim: Assess the effects of illness perception among psychiatric patients' level of internalized stigma as well as medication adherence. Subjects \& Method: Descriptive correlational design utilized with a total number of 191 patients who participated in the study. The Illness Perception Questionnaire Mental Health (IPQ-MH), Internalized Stigma of Mental Illness Inventory (ISMI), and Morisky Medication Adherence Scale (MMAS) were adopted to collect data. Results: patients appeared to have a high level of internalized stigma which is statistically correlated with illness perception. Concerning medication adherence, the results revealed that patients have a high level of compliance. Conclusion: Psychiatric patients vary in their perception of mental illness, some perceived it as a consequence of life stressors and others perceived it as a punishment. Further researches with a comprehensive study of patients' internalized stigma are extremely recommended. Recommendations: Educational campaigns for the public to mend and reform their conceptions and beliefs regarding mental illness also are required.
\end{abstract}

\section{Keywords : Illness perception, Internalized stigma, Medication adherence, Mental illness \& Psychiatric patients}

\section{Introduction}

When persons receive intimidations or immenseness regarding their health, they usually resort to evolve modalities of beliefs about this illness that commonly tamper and interpose their behaviors and emotions. These cognitive representations referred to the patient's perception of illness (Novitayani et al, 2020). In this context, Leventhal et al., 1982 developed a self regulatory model that reflects the patients' mental representations of their illnesses. They proposed that patients' perception of illness is processed through three stages: first is that individuals intellectually represent this perception, next is that they adopt behaviors to cope with it and finally, they speculate the leverage/ appraisal of these behaviors. The model also suggests that there is contentious feedback / reevaluation from the process of appraisal constantly influences patients' beliefs, emotional responses as well as coping styles. It proposed a causal relationship between illness cognitive beliefs and outcomes that is mediated by coping; this allows health providers to have an evident understanding of patients' behaviors and consequently helps them to enable patients to adjust appropriately with their illness process.

Further researches revealed that illness perception encompasses five components related to patients' set of beliefs (Leventhal \& Deifenbach, 1991); patients' beliefs about attributions of their illness (cause component), their beliefs regarding the nature and labeling of the disease as well as associated symptoms (identity component), conceptions related to whether their illness is acute, chronic or cyclic (timeline component), patient's beliefs about the extent to which their illness can be treated by therapy and controlled by their behaviors/ responses (curecontrol component), and finally, patients' beliefs about the impact of illness on their personal and social life as well as their perception of illness disability (consequences component) (Woon et al, 2020 \& Subramaniam et al, 2018).

Previous researches' results revealed that assessing illness perception has beneficial and profitable indicators across a wide range of common mental health disorders, such as schizophrenia (Tülay \& Funda, 2019; Subramaniam et al , 2018; Hussain et al., 2017), bipolar disorder (Averous, 2020; Novitayani et al., 2018) depression as well as anxiety (Scerri et al., 2019; Heer et al., 2017).

\section{Significance of the study}

One of the most experienced challenges for healthcare providers is the relapse of patients' condition particularly, psychotic patients. Diverse researches indicated that causes of psychotic relapse can be associated with stressful life events and lack of social support (Tülay \& Funda, 2019; Fikreyesus et al., 2016), chronicity of illness (Alphs et al., 2016), level of stigma, and medication non-compliance (Gathaiya et al., 2018). The extent to which patients perceive and cognitively represent their illness, affords healthcare providers references concerning how they can manage patients'disorders and how will be its' outcomes. In this context, profound studies 
revealed that perception of illness interferes with schizophrenic patients' quality of life and poor functioning (e.g. Sum, et al, 2020; Averous et al, 2018 \& Hussain et al, 2017;), depressed patients' low self esteem and increased severity of depressive symptoms (e.g. Nowicka-Sauer et al, 2018 \& Jaremo et al, 2017) and finally patients' medication adherence (e.g. Semahegn et al, 2018).

\section{Aim of the study}

Subsequently, this study aims to assess how mentally ill patients perceive their psychiatric illness and how this perception affects their level of internalized stigma as well as their adherence to medication. Moreover, the mediating effect of illness perception on the relationship between both internalized stigma and medication adherence levels will be also measured.

\section{Research question}

How psychiatric patients perceive their illness?

Does patients' illness perception affect level of their internalized stigma?

Does patients' illness perception affect level of their adherence to medication?

\section{Subjects \& Methods}

Study design and Sampling

Descriptive correlational design was utilized for this research. The study was conducted in out -patient clinics at the mental health hospital in Port-Said city. The hospital includes three out-patient clinics serving Port-Said governance and other near governances. Based on the following equation, (Vincent \& Thompson, 2014) the sample size was calculated:

$$
\mathrm{n}=\frac{\mathrm{NXp}(1-\mathrm{p})}{[\mathrm{N}-1(\mathrm{~d} 2 / \mathrm{z} 2)]+\mathrm{p}(1-\mathrm{p})}
$$

A total number of 191 patients participated in the study. Kaiser-Meyer-Olkin Measure of Sampling Adequacy test ( Glen, 2016) was done to ensure much reliability of this study's results (KMO test= .500). By following the simple randomizing technique, participants as well as their caregivers enrolled in the study, where the list of patients was formed based on patients' file number. Patients with the following criteria was excluded from the study, children diagnosed with child psychiatric disorders, mentally retarded patients, and patients during their acute phase of illness (as recommended by the study of Petrie et al, 2008.

The researcher started to collect data from May 2020 to August 2020 three days a week for about 5 hours daily. On the day of data collection, the researcher allocated a room specifically for data collection, to ensure confidentiality and communicate patients' sense of security. After explaining the aim of the study and mechanism of data collection to both patients and his/ her caregiver, and after guaranteed the confidentiality of data to be used only for research purposes for better treatment outcomes, the researcher asked the permission of the caregiver to interview the patient individually for the study purposes- to assure patient to communicate blankly and delightedly with no limits. Data was collected through the individual interview technique, in which every patient was individually interviewed for about $30-50$ minutes, according to patients' condition and response.

Some patients asked the researcher to fill in the questionnaires by themselves, thus the researcher assists the participants in the clarification of tools' instructions and sit beside patients for explaining any unclear statements for him/ her. Subsequently, the researcher thanked the patient and confirms anonymity and confidentiality of information obtained. The final step in data collection encompassed the researcher's reviewed all patients' files to collect basic clinical information and history of psychiatric illness.

Data collection was conducted after extraditing the issued ethical approval from the concerned administrative ethical boards and authorities (hospital administration; The General Secretariat of Mental Health. The research was also utilized in accordance with the Declaration of Helsinki and the ethical principles in the Belmont Report.

\section{Tools of data collection}

1. A structured questionnaire was developed by the researcher to collect socio-demographic and clinical data regarding patients as, age, sex, diagnosis and family history of psychiatric illness. Moreover, clinical history was also reviewed through patients' medical files.

2. The Illness Perception Questionnaire Mental Health (IPQ-MH) (Witteman et al, 2011), encompasses three parts. Part 1: the Identity scale consists of 12 items assessing patients' chief psychological complaints or problems. Part 2: the Structure scale includes 34 items with seven subscales-timeline chronic, timeline cyclical, consequences, personal control, treatment control, coherence and emotional representation subscales. Part 3: the Cause scale in which the psychological, biological, structural and stress-related causes are measured with 20 items.

Each statement is scored on five- point Likert scale ranging from $1=$ not important at all to $5=$ very much important. High scores induce the maladaptive perceptions of illness.

3. Internalized Stigma of Mental Illness Inventory(ISMI), includes29 items grouped into five subscales; Alienation; measured with six items and reflects patients' subjective experience 
of being less than others or having a 'spoiled identity'; Stereotype Endorsement, includes seven items revealing respondents' attitudes towards stereotypes related to mentally ill people; Perceived Discrimination, aims to scope/capture how participants perceive others' way in dealing with them assessed by and five statements. Finally, Social Withdrawal (6 items); and Stigma Resistance (5 items), intended to depict the experience of resisting or being unpretentious with internalized stigma. Each item is rated on four point Likert scale ranged from 1=strongly disagree to $4=$ strongly agree (Ritsher et al, 2003). The scoring system of the total scores is as follows: > 65 reflects low level of internalized stigma, 65-83 induced medium level, while scores ranging from 83-101 reflects high level of internalized stigma.

4. Morisky Medication Adherence Scale (MMAS). This self-report instrument contains eight questions discovering how frequently patients forgot to take their medications and their rationales. All questions were answered by Yes or No, except the final question (answered by never/ rarely/sometimes/often/always). The scoring interpretation for this scale is as follows: $0=$ reflects high adherence, 1-2= evolve medium adherence and scores less than 2, reflects low medication adherence (Morisky \& DiMatteo, 2011).

The researcher translated the tools into the local language (Arabic). The researcher adopted Beaton et al, 2000 guidelines for the process of cross-cultural adaptation of self-report measures, the forward and back translation done while a jury comprising five experts in psychiatric nursing reviews the translation for its consistency and content validity. Pretesting and cognitive debriefing were also utilized through a pilot study applied on $10 \%$ of patients to ensure the clarity of questions. The clinometric properties of the tools (IPQ-MH; ISMI; MMAS) were evaluated and results showed acceptable test-retest reliability, construct validity and internal consistency (Cronbach's Alpha = $0.88 ; 0.87 ; 0.89$ respectively).

\section{Statistical analysis}

Data retrieved from the utilized scales and questionnaires were coded and entered by using SPSS version 19. Demographic characteristics of respondents were represented in numbers, means and ranges and percentages according to data normal distribution. Moreover, descriptive analyses of the studied variables was also calculated and summed up and presented in numbers and percentages. Pearson inter subscales correlations were done to reflect the inter relationships among the studied variables. On the other hand, the researcher conducted a multivariable linear regression analysis to examine whether the independent variables (illness perception) portended each sub scores of the dependent variables (internalized stigma and medication adherence). Moreover, the mediating effect of illness perception on both internalized stigma and medication adherence was also measured by binary logistic analysis. Significance obtained at $\mathrm{p}$ value $<0.05$. 


\section{Results}

Table (1): Demographic characteristics of the studied psychiatric patients $(n=191)$.

\begin{tabular}{|c|c|c|}
\hline Socio-Demographic characteristics & Frequency $N=(191)$ & $\%$ \\
\hline \multicolumn{3}{|l|}{ Age $($ in years) } \\
\hline Less than 20 & 59 & 30.9 \\
\hline 20 - less than 25 & 45 & 23.6 \\
\hline 25 - less than 30 & 58 & 30.4 \\
\hline 30-less than 35 & 25 & 13.1 \\
\hline 35- less than 40 & 4 & 2.1 \\
\hline More than 40 & 59 & 30.9 \\
\hline \multicolumn{3}{|l|}{ Sex } \\
\hline Male & 103 & 53.9 \\
\hline Female & 88 & 46.1 \\
\hline \multicolumn{3}{|l|}{ Marital status } \\
\hline Single & 114 & 59.7 \\
\hline Married & 49 & 25.7 \\
\hline Divorced & 28 & 14.7 \\
\hline Widowed & 0 & 0.0 \\
\hline \multicolumn{3}{|l|}{ Level of education } \\
\hline Illiterate & 4 & 2.1 \\
\hline Primary & 30 & 15.7 \\
\hline Secondary/institute & 52 & 27.2 \\
\hline University & 105 & 55.0 \\
\hline \multicolumn{3}{|l|}{ Number of children } \\
\hline None & 111 & 58.1 \\
\hline $1-2$ & 70 & 36.6 \\
\hline $3-4$ & 10 & 5.2 \\
\hline$>4$ & 0 & 0.0 \\
\hline \multicolumn{3}{|l|}{ Occupation } \\
\hline None (male) & 86 & 45.0 \\
\hline House wife & 80 & 41.9 \\
\hline Practical / handmade / craft & 2 & 1.0 \\
\hline Technical Worker & 17 & 8.9 \\
\hline Professional & 6 & 3.1 \\
\hline Desk worker & 0 & 0.0 \\
\hline
\end{tabular}

Table (2): Clinical data of the studied psychiatric patients $(n=191)$.

\begin{tabular}{|l|c|c|}
\hline \multicolumn{1}{|c|}{ Clinical data } & Frequency N=(80) & \% \\
\hline Diagnosis & 58 & 30.4 \\
\hline Schizophrenia & 40 & 20.9 \\
\hline Major depression & 14 & 7.3 \\
\hline Mania & 23 & 12.0 \\
\hline Schizoaffective & 33 & 17.3 \\
\hline Anxiety disorders & 6 & 3.1 \\
\hline Somatoform disorders & 17 & 8.9 \\
\hline Personality disorders & 0 & 0.0 \\
\hline Others & & 29.3 \\
\hline Last hospitalization (months) & 56 & 50.3 \\
\hline None & 96 & 13.1 \\
\hline 1-3months & 25 & 7.3 \\
\hline 3-6 months & 14 & 0.0 \\
\hline 6-9 months & 0 & 0.0 \\
\hline 9-12 months & 0 & \multicolumn{2}{|c|}{} \\
\hline More than one year & \multicolumn{2}{|c|}{} \\
\hline
\end{tabular}




\begin{tabular}{|c|c|c|}
\hline siut Scientific Nursing Journal & & l-Sayad \\
\hline Clinical data & Frequency $\mathrm{N}=(\mathbf{8 0})$ & $\%$ \\
\hline \multicolumn{3}{|l|}{ Number of the previous hospitalization } \\
\hline None & 56 & 29.3 \\
\hline Once & 4 & 2.1 \\
\hline Two times & 15 & 7.9 \\
\hline Three times & 27 & 14.1 \\
\hline Four times & 39 & 20.4 \\
\hline More than 5 times & 50 & 26.2 \\
\hline \multicolumn{3}{|l|}{ Family history of mental illness } \\
\hline Yes & 96 & 50.3 \\
\hline No & 95 & 49.7 \\
\hline \multicolumn{3}{|c|}{ Did you ever change your employment due to mental illness? } \\
\hline Yes & 170 & 89.0 \\
\hline No & 21 & 11.0 \\
\hline \multicolumn{3}{|c|}{ Did you ever got separated/ divorced due to mental illness? lost social relationship } \\
\hline Yes & 191 & 100.0 \\
\hline No & 0 & 0.0 \\
\hline
\end{tabular}

Table (3): Illness perception subscales scores among the studied psychiatric patients $(n=191)$.

\begin{tabular}{|c|c|c|}
\hline 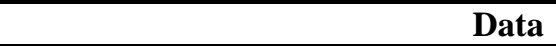 & Frequency & $\%$ \\
\hline \multicolumn{3}{|l|}{ Illness perception } \\
\hline \multicolumn{3}{|l|}{ Identity subscale } \\
\hline Mild & 0 & 0.0 \\
\hline Moderate & 33 & 17.3 \\
\hline High & 158 & 82.7 \\
\hline \multicolumn{3}{|l|}{ Structure subscale } \\
\hline Mild & 0 & 0.0 \\
\hline Moderate & 22 & 11.5 \\
\hline High & 169 & 88.5 \\
\hline \multicolumn{3}{|l|}{ Cause subscale } \\
\hline Mild & 0 & 0.0 \\
\hline Moderate & 42 & 22.0 \\
\hline High & 149 & 78.0 \\
\hline \multicolumn{3}{|l|}{ Total levels of illness perception } \\
\hline Moderate & 30 & 15.7 \\
\hline High (Maladaptive perception) & 161 & 84.3 \\
\hline
\end{tabular}

Table (4): Internalized stigma subscales scores and levels among the studied psychiatric patients $(\mathbf{n}=191)$.

\begin{tabular}{|c|c|c|}
\hline Data & Frequency & $\%$ \\
\hline \multicolumn{3}{|c|}{ Internalized Stigma of mental illness subscales: } \\
\hline \multicolumn{3}{|c|}{ Alienation } \\
\hline Moderate (12-18) & 31 & 16.2 \\
\hline High $(18-24)$ & 160 & 83.8 \\
\hline \multicolumn{3}{|l|}{ Stereotype Endorsement } \\
\hline Moderate (14-21) & 25 & 13.1 \\
\hline High (21-28) & 166 & 86.9 \\
\hline \multicolumn{3}{|l|}{ Discrimination Experience } \\
\hline Moderate (10-15) & 43 & 22.5 \\
\hline High $(15-20)$ & 148 & 77.5 \\
\hline \multicolumn{3}{|l|}{ Social Withdrawal } \\
\hline Moderate (12-18) & 27 & 14.1 \\
\hline High $(18-24)$ & 164 & 85.9 \\
\hline
\end{tabular}




\begin{tabular}{|l|c|c|}
\hline Stigma Resistance & 156 & 81.7 \\
\hline Moderate (15-20) & 35 & 18.3 \\
\hline High (15-10) & \multicolumn{3}{|l|}{} \\
\hline Total level of internalized stigma & 24 & 12.6 \\
\hline Moderate (65-83) & 167 & 87.4 \\
\hline High (83-101)
\end{tabular}

Table (5): Medication adherence level of the psychiatric patients

\begin{tabular}{|c|c|c|}
\hline Data & Frequency & $\%$ \\
\hline \multicolumn{3}{|c|}{ Q1: Do you sometimes forget to take your medications? } \\
\hline Yes & 63 & 33.0 \\
\hline No & 128 & 67.0 \\
\hline \multicolumn{3}{|c|}{$\begin{array}{l}\text { Q2: People sometimes miss taking their medications for reasons other than forgetting. Thinking ove } \\
\text { the past two weeks, were there any days when you did not take your medications? }\end{array}$} \\
\hline Yes & 63 & 33.0 \\
\hline No & 128 & 67.0 \\
\hline \multicolumn{3}{|c|}{$\begin{array}{l}\text { Q3: Have you ever cut back or stopped taking your medications without telling your doctor, because } \\
\text { you felt worse when you took it? }\end{array}$} \\
\hline Yes & 63 & 33.0 \\
\hline No & 128 & 67.0 \\
\hline \multicolumn{3}{|c|}{ Q4: When you travel or leave home, do you sometimes forget to bring along your medications? } \\
\hline Yes & 63 & 33.0 \\
\hline No & 128 & 67.0 \\
\hline \multicolumn{3}{|c|}{ Q5: Did you take your medications yesterday? } \\
\hline Yes & 128 & 67.0 \\
\hline No & 63 & 33.0 \\
\hline \multicolumn{3}{|c|}{$\begin{array}{l}\text { Q6: When you feel like your health condition is under control, do you sometimes stop taking you } \\
\text { medications? }\end{array}$} \\
\hline Yes & 63 & 33.0 \\
\hline No & 128 & 67.0 \\
\hline \multicolumn{3}{|c|}{$\begin{array}{l}\text { Q7: Taking medications every day is a real inconvenience for some people. Do you ever feel hasslec } \\
\text { about sticking to your treatment plan? }\end{array}$} \\
\hline Yes & 63 & 33.0 \\
\hline No & 128 & 67.0 \\
\hline \multicolumn{3}{|c|}{ Q8: How often do you have difficulty remembering to take all your medications? } \\
\hline Never/rarely & 64 & 33.5 \\
\hline Once in a while & 73 & 38.2 \\
\hline Sometimes & 3 & 1.6 \\
\hline Usually & 51 & 26.7 \\
\hline All the time & 0 & 0.0 \\
\hline \multicolumn{3}{|c|}{ Total score of level of medication adherence } \\
\hline Low $(8-12)$ & 54 & 28.3 \\
\hline $\operatorname{High}(16-17)$ & 137 & 71.7 \\
\hline
\end{tabular}


Table (6): Correlation among the illness perception, internalized stigma and medication adherence among the studied patients.

\begin{tabular}{|c|c|c|c|c|c|c|c|c|c|c|c|}
\hline & 1 & 2 & 3 & 4 & 5 & 6 & 7 & 8 & 9 & 10 & 11 \\
\hline Identity subscale (IPQ-MH) & - & & & & & & & & & & \\
\hline $\begin{array}{l}\text { Structure subscale (IPQ- } \\
\mathrm{MH})\end{array}$ & $\begin{array}{c}.906 \\
(.009)^{* *}\end{array}$ & & & & & & & & & & \\
\hline Cause subscale (IPQ-MH) & $\begin{array}{c}.423 \\
(.058)^{*}\end{array}$ & $\begin{array}{c}.93 \\
(.006)^{* *}\end{array}$ & & & & & & & & & \\
\hline $\begin{array}{l}\text { Total score of illness } \\
\text { perception }\end{array}$ & $\begin{array}{l}-.083 \\
(.253)\end{array}$ & $\begin{array}{l}-.111 \\
(.127)\end{array}$ & $\begin{array}{c}.188 \\
.009 * *\end{array}$ & & & & & & & & \\
\hline Alienation (ISMI) & $\begin{array}{l}.740 \\
(.024)^{*}\end{array}$ & $\begin{array}{c}.793 \\
(.019)^{* *}\end{array}$ & $\begin{array}{l}-.091 \\
(.184)\end{array}$ & $\begin{array}{c}.944 \\
(.005)^{*}\end{array}$ & & & & & & & \\
\hline $\begin{array}{l}\text { Stereotype Endorsement } \\
\text { (ISMI) }\end{array}$ & $\begin{array}{l}.130 \\
(.110)\end{array}$ & $\begin{array}{c}.454 \\
(.054)^{*}\end{array}$ & $\begin{array}{l}.070 \\
(.131)\end{array}$ & $\begin{array}{l}-.125 \\
(.085)\end{array}$ & $\begin{array}{l}-.125 \\
(.088)\end{array}$ & & & & & & \\
\hline $\begin{array}{l}\text { Discrimination Experience } \\
\text { (ISMI) }\end{array}$ & $\begin{array}{l}-.081 \\
(.268) \\
\end{array}$ & $\begin{array}{l}-.037 \\
(.607)\end{array}$ & $\begin{array}{l}-.014 \\
(.850)\end{array}$ & $\begin{array}{c}-.06 \\
(.406) \\
\end{array}$ & $\begin{array}{l}-.101 \\
(.163) \\
\end{array}$ & $\begin{array}{c}.484 \\
(.051)^{*}\end{array}$ & & & & & \\
\hline Social Withdrawal (ISMI) & $\begin{array}{c}-.145 \\
(.106)^{* *}\end{array}$ & $\begin{array}{c}-.565 \\
(.106)^{* *}\end{array}$ & $\begin{array}{c}.975 \\
(.002)^{* *}\end{array}$ & $\begin{array}{l}.318 \\
(.073)\end{array}$ & $\begin{array}{l}.365 \\
(.066)\end{array}$ & $\begin{array}{l}-.744 \\
(.024)^{*}\end{array}$ & $\begin{array}{l}-.304 \\
(.075)\end{array}$ & & & & \\
\hline Stigma Resistance (ISMI) & $\begin{array}{c}.982 \\
(.002)^{* *}\end{array}$ & $\begin{array}{c}.548 \\
(.044)^{*}\end{array}$ & $\begin{array}{l}.755 \\
(.23)\end{array}$ & $\begin{array}{c}-.797 \\
(.019) * *\end{array}$ & $\begin{array}{l}.176 \\
(.098)\end{array}$ & $\begin{array}{l}-.182 \\
(.097)\end{array}$ & $\begin{array}{l}.158 \\
(.029)^{*}\end{array}$ & $\begin{array}{c}-.978 \\
(.002)^{* *}\end{array}$ & & & \\
\hline $\begin{array}{l}\text { Total level of Internalized } \\
\text { Stigma }\end{array}$ & $\begin{array}{l}.119 \\
(.100)\end{array}$ & $\begin{array}{c}.873 \\
(.012)^{* *}\end{array}$ & $\begin{array}{c}.503 \\
(.009)^{* *}\end{array}$ & $\begin{array}{c}.951 \\
(.004)^{* *}\end{array}$ & $\begin{array}{l}.891 \\
(.010)\end{array}$ & $\begin{array}{c}.181 \\
(.012)^{* *}\end{array}$ & $\begin{array}{l}-.053 \\
(.466)^{*}\end{array}$ & $\begin{array}{l}-.108 \\
(.035)\end{array}$ & $\begin{array}{l}.144 \\
(-.106)\end{array}$ & & \\
\hline $\begin{array}{l}\text { Total level of Medication } \\
\text { Adherence (MMAS) }\end{array}$ & $\begin{array}{c}.010 \\
(-.889)\end{array}$ & $\begin{array}{l}.028 \\
(.696)\end{array}$ & $\begin{array}{l}.017 \\
(.172)\end{array}$ & $\begin{array}{c}.268 \\
(.080)\end{array}$ & $\begin{array}{c}.231 \\
(-.087)\end{array}$ & $\begin{array}{c}-.002 \\
(.974)\end{array}$ & $\begin{array}{l}.014 \\
(.952)\end{array}$ & $\begin{array}{l}.055 \\
(.454)\end{array}$ & $\begin{array}{c}.087 \\
(.231)\end{array}$ & $\begin{array}{l}.285 \\
(.078)\end{array}$ & - \\
\hline
\end{tabular}

Pearson correlation test

*Correlation is significant at the 0.05 level (2-tailed)

** Correlation is significant at the 0.01 level (2-tailed)

Note: (IPQ-MH): Illness Perception Questionnaire Mental Health; (ISMI): Internalized Stigma of Mental Illness Inventory; (MMAS): Morisky Medication

Adherence Scale 
Table (7): Logistic regression analysis of the significant correlates with illness perception

\begin{tabular}{|c|c|c|c|c|c|c|c|}
\hline Variables & B & SE & Wald & P-Value & $\mathbf{O R}$ & 95\% CI (Lower) & 95\% CI (Upper) \\
\hline Identity subscale (IPQ-MH) & -1.396 & .977 & 2.042 & .153 & .248 & .036 & 1.680 \\
\hline Structure subscale (IPQ-MH) & -1.489 & 1.131 & 1.735 & .188 & .226 & .025 & 2.069 \\
\hline Cause subscale (IPQ-MH) & 1.532 & .540 & 8.052 & $.043 *$ & 4.628 & 1.606 & 13.335 \\
\hline Alienation (ISMI) & .445 & .658 & .458 & .499 & 1.561 & .430 & 5.668 \\
\hline Stereotype Endorsement (ISMI) & -2.046 & 1.149 & 3.169 & .075 & .129 & .014 & 1.229 \\
\hline Discrimination Experience (ISMI) & -.337 & .618 & .297 & .585 & .714 & .213 & 2.396 \\
\hline Social Withdrawal (ISMI) & .027 & 659 & .002 & .967 & 1.028 & .282 & 3.739 \\
\hline Stigma Resistance (ISMI) & -.593 & .625 & .898 & .343 & .553 & .162 & 1.883 \\
\hline Total level of Internalized Stigma & .971 & .735 & 1.745 & $.001 * *$ & 2.641 & .625 & 11.159 \\
\hline Total level of Medication Adherence (MMAS) & .071 & 1.717 & .002 & .753 & 1.073 & .037 & 31.078 \\
\hline Constant & 3.972 & 8.104 & .240 & .624 & 53.116 & & \\
\hline
\end{tabular}

*Statistically significant at $\mathrm{P} \leq 0.05$

** Highly statistically significant $\mathrm{P} \leq 0.001$.

Model was controlled for the following demographic as well as clinical data of the participants

Abbreviations: B, beta coefficient; SE, standard error; OR, odds ratio; CI, confidence interval (IPQ-MH): Illness Perception Questionnaire Mental Health; (ISMI):

Internalized Stigma of Mental Illness Inventory; (MMAS): Morisky Medication Adherence Scale

Table (8): Mediation effect of Illness perception on the relationship between internalized stigma and medication adherence

\begin{tabular}{|c|c|c|c|c|c|c|c|c|}
\hline $\begin{array}{c}\text { Regression } \\
\text { model }\end{array}$ & Dependant Variables & $\begin{array}{c}\text { Independent } \\
\text { Variables } \\
\end{array}$ & B & SE & Wald & P-Value & $\mathbf{O R}$ & $95 \% \mathrm{CI}$ \\
\hline 1. & Internalized Stigma & Illness perception & .727 & .620 & .134 & $.003 * *$ & 1.255 & $(0.372-1.824)$ \\
\hline 2. & Internalized Stigma & $\begin{array}{l}\text { Medication } \\
\text { Adherence }\end{array}$ & -.351 & .501 & .490 & $.054 *$ & 0.420 & $(0.532-1.826)$ \\
\hline 3. & Medication Adherence & Illness perception & -.511 & .451 & 1.283 & .447 & 0.668 & $(0.688-9.040)$ \\
\hline 4. & $\begin{array}{l}\text { Medication Adherence Internalized } \\
\text { Stigma }\end{array}$ & Illness perception & .266 & .498 & .540 & .214 & 1.441 & $(0.544-4.040)$ \\
\hline
\end{tabular}

Sobel test: $\mathrm{Z}=2.053, \mathrm{P}=.3251$

* Statistically significant at $\mathrm{P} \leq 0.05$

** Highly statistically significant $\mathrm{P} \leq 0.001$

Abbreviations: B, beta coefficient; SE, standard error; OR, odds ratio; CI, confidence interval 
Demographic \& Clinical data characteristics

Patients' age was ranged between 17- 49 years; with the highest percentage $(30.9 \%)$ showed in patients aged more than 40 years as well as patients aged less than 20 years. More than half of the patients were males and had university level of education $(53.9 \%, 55.0 \%$ respectively). Furthermore, table 1 shows that almost half of the male patients $(45.0 \%)$ had no work as compared to $41.9 \%$ was female patients.

Results also revealed that, about one third of patients $(30.4 \%)$ were diagnosed with schizophrenia compared to $20.9 \%$ diagnosed with major depression and $17.3 \%$ with anxiety disorders. Half of the patients $(50.3 \%)$ had their last hospitalization from 1-3 months prior to the study and $29.3 \%$ reported to having no previous history of hospitalization; moreover, $50.3 \%$ of them mentioned that they had a family history of mental illness. Finally, all of the patients reported that their illness caused them to lose some of their social relationships (e.g. divorced or separated from spouse, distance from close friends or relatives) as compared to $89.0 \%$ who stated that they lost their work because of their mental illness. Table 2.

Descriptive analysis of illness perception, internalized stigma and medication adherence

Table (3): Reveals illness perception among patients. Results induced high scores about identity, structure and cause subscales $(82.7 \%$, $88.5 \%$, and $78.0 \%$ respectively); where the total scores of a high level of illness perception were 84.3\%; reflecting maladaptive perception. Most symptoms reported by patients were cognitive complaints, behavioral symptoms, sleeping problems and somatic complaints respectively. The studied patients related these complaints to their social circumstances, their life style and their illness.

Concerning the structure subscale, most the patients believed that their psychiatric illness will be permanent and will last for the rest of their lives, and that their illness symptoms are usually fluctuating over time. Moreover, most of the respondents mentioned that mental illness has major consequences on their lives and strongly affects others' attitudes. Furthermore, most of the patients stated that they have no control over their illness but the treatment does. Patients' responses related to coherence reflected that their illness masters their lives with a clear understanding. Finally, most patients stated that their illness makes them feel depressed, worried and angry.

The final subscale of the illness perception scale represents causes of mental illness as perceived by the respondents. Most of the patients said that their illness is mainly related to life stressors (e.g. conflicts with a partner, recent unpleasant events, lack of coping mechanisms) as well as family relationships, unresolved conflicts and hereditary factors.

As regards internalized stigma, table 4 shows that more than two thirds of patients $(87.4 \%)$ experienced internalized stigma, with the highest percentages $(86.9 \%)$ linked with stereotype endorsement, followed by social withdrawal, alienation and discrimination experience $(85.9 \%$, 83.8\%,77.5\%). Concerning patients' adherence to medication, Table 5 clarifies that $71.7 \%$ of respondents were found to be highly comply with their medication.

Correlation \& Regression analysis of the studied variables

Table (6): Illustrates correlation analysis among scales and the subscales of the studied variables. As the table clarifies, a significant positive correlation found between illness perception and internalized stigma while no correlation was observed about medication adherence with these two variables.

For more interpretation of data, multiple linear regression analyses were performed to explore correlates of illness perception among the participants. As table 7 represents, that illness perception level was the highly significant predictor of patients' internalized stigma level, but not for medication adherence level $(\mathrm{P}=.001$, $\mathrm{P}=.753$ respectively). Based on these results, the researcher tested the mediating effect of illness perception on the relationship between medication adherence and internalized stigma, and clarified in table 8 that when illness perception was added as a mediator predicting high internalized stigma level, the relationship between internalized stigma and medication adherence levels was not significantly mediated $(\mathrm{OR}=1.441,95 \% \mathrm{CI}=0.544-4.040)$, where Sobel test also confirmed this result $(\mathrm{Z}=2.053$, $\mathrm{P}=.3251)$.

\section{Discussion}

This study aimed to assessing how mentally ill patients perceive their psychiatric illness and how this perception affects their level of internalized stigma as well as their medication adherence. Concerning illness perception, results induced a high level of identity scores which reveals patients' perception of symptoms as well as the attributions of these symptoms. Patients related it to their social stressors, poor economical status as well as family miscommunication patterns. In the same context, psychiatric patients perceived their symptoms to last to the rest of their lives but its severity often oscillates over days, and their treatment is the key to recovery in which they have no control. The researcher also observed pessimism in patients' tone of voice and facial expressions and 
one female patient cried while stating "I feel I will be sick all my life, once I feel I am better, I returned again sick, why God always punish me. Why me?". The fact that psychiatric illness is chronic should be communicated empathetically and rationally with patients.

In this context, almost all of the studied patients proclaimed that mental illness sorely and grudgingly affected their lives, particularly social relationships. All of the studied patients reported to be divorced or separated from their partners as a consequence of their illness; furthermore, one male patient stated "after hospitalization for three months, I went to my work but my manager was surprised and looked afraid to talk to me, he straighten up of his office desk and stood beside the door and spoke cautiously that I am in open vacation till he will call". Another female patient stated "after my delivery of my child, I diagnosed with postpartum depression, when my husband hears that, he said you are not eligible now to be a mother or a wife. Go and stay in the hospital it is better for all of us. And he took my son away from me". Truly, public negative misconceptions about mental illness regulate their behaviors and attitudes which consequently influence patients' social lives and treatment period. This result is supported by the studies of Philip et al, 2020; Sum et al., 2020; Wang et al., 2017; Yilmaz \& Okanli, 2016.

Most of the patients related mental disorders to life stressors, conflicts and as a consequence of their lifestyles respectively. Some of the patients predominantly in late teens to late thirteen bind their illness to economic factors and poverty. One of the patients said "you know why I do not want to take my medication, how come that a young person spends his life in the bed. Medication always let me sleep all the time. If I slept in my youth, what I will do when I grow up?" Similar result reported in the study of Pingali et al, 2018 who conducted a comparative study of the internalized stigma and its correlates among different psychiatric disorders in remission, they reported that patients conceived mental illness to be a punishment for their negligence of religious rituals, impetuous and reckless acts as well as irreverent personality. In the same context, other studies (e.g. Averous et al, 2020; Tülay \& Funda, 2019; Hussain et al., 2017; \& Jaremo et al., 2017) stated that patients' illness perception was highly correlated with life stressors and conflicts.

Concerning internalized stigma, the results of the present study revealed that more than two thirds of patients had a high level. Expectedly on the light of the previous results and patients' statements, most of patients experienced internalized stigma. Regrettably, media still communicate fallacies and misconceptions associated with mental illness, and as a consequence, people communicate these stereotypical faulty ideas to patients and their caregivers. Moreover, patients tend to internalize them and translate it into negative self talk. Most of the participants in this study felt inferior to others, ashamed to state that they are mentally ill and depend on others especially in making decisions. One patient said "my wife told me that I should not sit with our visitors because they will know that I am sick when they saw my face and she does not want to let anyone knows". This result is consistent with the diverse studies (e.g. Kaşli et al, 2020; Szcześniak et al., 2018; Turkmen et al., 2018; Pearl, Forgeard \& Rifkin et al., 2017)

Furthermore, patients reported that they are highly discriminated from others only because they are mentally ill. Indeed, this result can be correlated with previous results concerning losing relationships and work as a consequence of mental illness. Moreover, people still perceive mental patients as unproductive, worthless and inutile persons. This result is in line with the studies of Alemayehu, 2020; Yildirim, Kavak, 2020; Sy-Cherng et al, 2020; \& GonzálezDomínguez et al., 2019; who pronounced in their studies that the level of stigma was highly correlated with poor social support, poor quality of life and being single. In this context some patients in the present study reported "when my wife knows that I am mentally ill before our marriage, she told me that we should not have any children and when I arguing her she told me you are not the rational father you will cause danger to your children if they become inherited with mental illness. You want them to live a miserable life like you?" .Patients will not only tolerate their illness, rather they will also endure others' problems.

The results of this study reflected that more than half of the patients comply with their treatment. Patients as well as their caregivers were keen to follow up and prescribed therapies, patients reported that they need medication in order to not hospitalize again and caregivers forced the patient to adhere to medication to control symptoms. On the other hand, some of the patients pronounced that medication is useless; and that it is only narcotics, and other patients claimed that they do not believe in psychiatric treatment as they perceive their problems linked with mainly life stressors, thus if they changed it they will be recovered. On this context, a patient declared "I read a lot about my mental illness and I believed that it is a life time illness with no cure, medications are prescribed only to let me calm down and sleep in order to not produce any problems with my family". Another patient announced "I do not need medication, my actual 
problem is my mom's style of communication; if she simply changes it I will be fine". This result is in line with the results of Semahegn et al., 2020 who studied the determinants of medication adherence among psychiatric patients and found that more than half of them were adherent to their medication, but this is not consistent with the results of Dushad et al., 2019; Lee, Lin, Chang, Shao, 2018 \& Semahegn et al., 2018.

Eventually, although patients' perception of their psychiatric illness seemed to be a significant predictor of internalized stigma level it does not mediates the relationship between internalized stigma and medication adherence levels. This result may be due to that misperception of illness which is reflected by the participants in this study, which direct them towards internalization of negative thoughts that they are inferior, irresponsible, dependant, and discriminated as well as rejected by others. This result was not supported by the study of Abdisa et al., 2020 who stated in their study that self stigma was negatively associated with medication adherence among psychiatric patients. Moreover, Pribadi et al., 2020 also discovered the negative impact of internalized stigma on patients' adherence and attitudes towards medication.

\section{Conclusion}

Based on this study results, psychiatric patients vary in their perception of mental illness, some perceived it as a consequence of life stressors and others perceived it as a punishment. Moreover, most patients stated that they lost their job or their social/ intimate relationships as a result of their mental disorders. On the other hand, participants appeared to have a high level of internalized stigma which is statistically and positively correlated with illness perception. Concerning medication adherence, the results revealed that patients have a high level of compliance. Furthermore, illness perception significantly mediated the relationship between internalized stigma and medication adherence levels.

Implications of the study \&
Recommendations

Further researches with a comprehensive study of patients' internalized stigma are extremely recommended. Stigma indeed affects patients' perception of illness as a burden, thus compiling a program for patients as well as their caregivers would be profitable in emending and rectifying misconceptions concerning mentally ill persons. Educational campaigns for the public to mend and reform their conceptions and beliefs regarding mental illness are also suggested.

Conflict of interest

No conflict pronounced by the researcher Fund
None

\section{References}

- Abdisa, E., Fekadu, G., Girma, S., Shibiru, T., Tilahun, T., Mohamed, H., Wakgari, A., Takele, A., Abebe, M., \& Tsegaye, R. (2020): Self-stigma and medication adherence among patients with mental illness treated at Jimma University Medical Center, Southwest Ethiopia. International journal of mental health systems, 14, https://doi.org/10.1186/s13033-020-00391-6

- Alemayehu, Y. (2020): Internalized Stigma and Associated Factors among Patients with Major Depressive Disorder at the Outpatient Department of Amanuel Mental Specialized Hospital, Addis Ababa, Ethiopia, 2019: A Cross-Sectional Study. Psychiatry Journal. Article https://doi.org/10.1155/2020/7369542

- Alphs, L., Henry, N., Cynthia, B., DongJing, F., Srihari, G., David, H., \& Ibrahim, T. (2016): Factors associated with relapse in schizophrenia despite adherence to long-acting injectable antipsychotic therapy. International Clinical Psychopharmacology, 31(4):202-209. doi: 10.1097/YIC.0000000000000125.

- Averous P, Charbonnier E, LagouanelleSimeoni M-C, \& Dany L. (2018): Illness representations about schizophrenia and bipolar disorder held by French people without a mental disorder. International Journal of Social Psychiatry; 64(8):813-820. doi:10.1177/0020764018814272

- Averous P, Charbonnier E, \& Dany L. (2020): Relationship between Illness Representations, Psychosocial Adjustment, and Treatment Outcomes in Mental Disorders: A Mini Review. Frontier in Psychology, 12 June 2020. Available at: https://www.frontiersin.org/ articles/10.3389/ fpsyg.2020.01167/full

- Dushad, R., Mintu, M., Samaksha, P., \& Basavana, G. (2019): A study of drug attitude and medication adherence and its relationship with the impact of illness among the mentally ill. Archives of Clinical Psychiatry (São Paulo), 46(4), 85-88. Epub August 29, 2019.https://dx.doi.org/10.1590/010160830000000201

- Fikreyesus, M., Soboka, M., \& Tolu Feyissa, G. (2016): Psychotic relapse and associated factors among patients attending health services in Southwest Ethiopia: a crosssectional study. BMC Psychiatry, 16:354- 377.

- Gathaiya, N, Mwaura, J., Wagoro, M, (2018): A Cross-Sectional Study on Factors Associated with Relapse in Patients with Schizophrenia at Mathari Hospital, Nairobi, Kenya. Annals of Clinical and Laboratory 
Research, 6(1):218. DOI: 10.21767/23865180.1000218.

- Glen, S. (2016): "Kaiser-Meyer-Olkin (KMO) Test for Sampling Adequacy" From Statistics: Elementary Statistics for the rest of us!. Available

https://www.statisticshowto.com/kaisermeyer-olkin/

- González-Domínguez, S., GonzálezSanguino, C., \& Muñoz, M. (2019): Efficacy of a combined intervention program for the reduction of internalized stigma in people with severe mental illness. Schizophrenia Research, Sep 211: 56-62. Available at: https://pubmed.ncbi.nlm.nih.gov/31351829/

- Heer, E., Vriezekolk, J., van der FeltzCornelis, C. (2017): Poor Illness Perceptions Are a Risk Factor for Depressive and Anxious Symptomatology in Fibromyalgia Syndrome: A Longitudinal Cohort Study. Frontiers Psychology, $8: 217$. doi:10.3389/fpsyt.2017.0021. https://doi.org/10.1111/jpm.12611

- Hussain, S., Imran, N., Hotiana, U., Mazhar, N., \& Asif, A. (2017): Illness Perceptions in Patients of Schizophrenia: A Preliminary Investigation from Lahore, Pakistan. Pakistan journal of medical sciences, 33(4), 829-834. https://doi.org/10.12669/pjms.334.13128

- Jaremo, P., Arman, M., Gerdle, B., Larsson, B., \& Gottberg, K. (2017): Illness beliefs among patients with chronic widespread pain associations with self-reported health status, anxiety and depressive symptoms and impact of pain. BMC Psychol 5(1), 24. doi: 10.1186/s40359-017-0192-1.

- Kaşli, O., \& Bademli, K. (2020): Internalized stigmatization and subjective recovery in individuals with chronic mental illness. International Journal of Social Psychiatry. https://doi.org/10.1177/002076402 0960762

- Lee, SK., Lin, E., Chang, YF., WC. \& Shao, R. (2018): Psychometric Evaluation of Family Illness Perceptions of Relatives with Schizophrenia. Neuropsychiatry (London), 8(1): 102- 110. Available at: https://www.jneuropsychiatry.org/abstract/psyc hometric-evaluation-of-family-illnessperceptions-of-relatives-with-schizophrenia12352.html

- Leventhal H., \& Diefenbach M. (1991): The Active Side of Illness Cognition. In: Skelton J.A., Croyle R.T. (eds) Mental Representation in Health and Illness. Contributions to Psychology and Medicine. Springer, New York, NY.

- Leventhal, H., Nerenz., D,R., \& Steele, D,J. (1982): Illness Representations and Coping with Health Threats. Handbook of psychology and health, volume IV: social psychology aspects of health. Hillsdale. NJ:Erlbaum

- Morisky D., \& DiMatteo M. (2011): Improving the measurement of self-reported medication non-adherence: final response. Journal of Clinical. Epidemiology. 64, 255-263. Available at: https://www.ncbi.nlm.nih.gov/pmc/articles/PM C3109729/

- Novitayani, S., Chanchong, W., \& Suttharangsee, W. (2020): Illness Perception of Schizophrenia among Schizophrenic Outpatients. In Proceedings of the Aceh International Nursing Conference: 242-249. DOI: 10.5220/0008397002420249.

- Nowicka-Sauer K, Hajduk A, KujawskaDanecka H, Banaszkiewicz D, Smoleńska $\dot{Z}$, Czuszyńska Z, \& Siebert J. (2018): Illness perception is significantly determined by depression and anxiety in systemic lupus erythematosus. Lupus. 27(3):454-460. doi: 10.1177/0961203317751858. Epub 2018 Jan 11

- Pearl, R., Forgeard, M., Rifkin, L., Beard, C., \& Björgvinsson, T. (2017): Internalized stigma of mental illness: Changes and associations with treatment outcomes. Stigma and Health, 2(1), 215. https://doi.org/10.1037/sah0000036

- Petrie, K. J., Broadbent, E., Kydd, R. (2008). Illness perceptions in mental health: Issues and potential applications. Journal of Mental Health, 17(6), 559564. https://doi.org/10.1080/096382308025230 47

- Philip T. Yanos, Joseph S. DeLuca, David Roe, Paul H. \& Lysaker. (2020): The Impact of Illness Identity on Recovery from Severe Mental Illness: A Review of the Evidence, Psychiatry Research, 10.1016/j.psychres.2020.112950. Available at: https://pubmed.ncbi.nlm.nih.gov/32361335/

- Pingali, S., Srinivasa, S., Umashankar, M. (2018): A comparative study of internalized stigma and its correlates among different psychiatric disorders in remission. Telangana Journal of Psychiatry, 4(1):29-33. Available at: https://www.ipinnovative.com/journal-articlefile/6966

- Pribadi, T., Lin, E., Chen, P, Lee, S, Fitrayasari, R., \& Chen, C, (2020): Factors associated with internalized stigma for Indonesian individuals diagnosed with schizophrenia in a community setting. Journal of Psychiatric and Mental Health Nursing, 27 (5): 584-594. Available at: https://pubmed.ncbi.nlm.nih.gov/32009270/

- Ritsher, JB., Otilingam, G., \& Grajales, M. (2003): Internalized stigma of mental illness: psychometric properties of a new 
measure. Psychiatry Research; 121(1):31-49. Available at:

https://pubmed.ncbi.nlm.nih.gov/14572622/

- Scerri, J., Saliba, T., Saliba, G., Scerri C., \& Camilleri, L. (2019): Illness perceptions, depression and anxiety in informal carers of persons with depression: a cross-sectional survey. Quality of Life Research, 28: 451- 460 https://doi.org/10.1007/s11136-018-2009-y.

- Semahegn, A., Torpey, K., Manu, A., Assefa, N., Tesfaye, G., \& Ankomah, A. (2018): Psychotropic medication non-adherence and associated factors among adult patients with major psychiatric disorders: a protocol for a systematic review. Systematic reviews, 7(1), 10. doi:10.1186/s13643-018-0676-y

- Subramaniam., M, Abdina., E, Jeyagurunathana., A, Changa., S, Samaria., E, Shafiea., S, Weib., KC, Vermac., S, \& Chonga., SA. (2018): Exploration of illness perception among patients with mental illness in a multi-ethnic Asian sample. Psychiatry Research, 267:516- 527. Available at: https://pubmed.ncbi.nlm.nih.gov/29980132/

- Sum, M, Chan, S, Tse, S., Bola, J., Ng, R., Hui, K, Lee, E., Chang, W, \& Chen, E, (2020): Elucidating the relationship between internalized stigma, cognitive insight, illness severity, and functioning in patients with schizophrenia using a path analysis approach, Journal of Mental Health, 10.1080/09638237.2020.1836553, (1-

10). Available at:

https://pubmed.ncbi.nlm.nih.gov/33095070/

- Sy-Cherng, W, Im, K, Azlin, B., \& Marhani, M. (2020): Association between insight and internalized stigma and other clinical factors among patients with depression: A crosssectional study. Indian Journal of Psychiatry. 62(2): 186-192. Available at: https://www.ncbi.nlm.nih.gov/pmc/articles/PM C7197844/

- Szcześniak, D., Kobyłko, A., Wojciechowska, I., Klapciński, A., \& Rymaszewska, J. (2018): Internalized stigma and its correlates among patients with severe mental illness.Neuropsychiatric Disorders Treatment; 14: 2599-2608. doi: 10.2147/NDT.S169051

- Tülay, Y., \& Funda, B. (2019): The relationship between internalized stigma and loneliness in patients with schizophrenia. Perspectives in Psychiatric Care, 10.1111/ppc.12399, 56, 1:168-174.

Available at: https://pubmed.ncbi.nlm.nih.gov/31093994/

- Turkmen, S., Yorulmaz, M., Koza, E., \& Ozdemir, S. (2018): Internalized stigmatization and social functioning in psychiatric patients. Journal of Turgut Ozal
Medical Centere, 25(1):12-7. DOI: 10.5455/jtomc.08.116

- Vincent, K., \& Thompson, S. (2014): Estimating Population Size with Link-Tracing Sampling. Retrieved from: https://arxiv.org/pdf/1210.2667.pdf

- Wang, Q., Petrini, B., \& Morisky E. (2017): Predictors of quality of life among Chinese people with schizophrenia. Nursing Health Sciences, $19 \quad$ (2):142-148. doi: 10.1111/nhs.12286. Epub 2017. Apr 25. PMID: 27109014. Available at: https://pubmed.ncbi.nlm.nih.gov/27109014/

- Witteman, C., Bolks, L., \& Hutschemaekers, G. (2011): Development of the illness perception questionnaire mental health. Journal of Mental health, 20(2): 115- 125. Available at: $\quad$ https://www.sciesocialcareonline.org.uk/development-of-theillness-perception-questionnaire-mentalhealth/r/a1CG0000000GLy2MAG

- Woon, L, Khoo, S, Baharudin, A., \& Midin, M. (2020): Association between insight and internalized stigma and other clinical factors among patients with depression: A crosssectional study. Indian Journal of Psychiatry, 62:186-92. Available at: https://pubmed.ncbi.nlm.nih.gov/32382179/

- Yildirim, T., \& Kavak, F. (2020): The relationship between internalized stigma and loneliness in patients with schizophrenia. Perspective Psychiatric Care. Jan;56 (1):168174. doi: 10.1111/ppc.12399. Epub 2019 May 15. PMID: 31093994. Available at: https://pubmed.ncbi.nlm.nih.gov/31093994/

- Yılmaz E, \& Okanlı A. (2015): The Effect of Internalized Stigma on the Adherence to Treatment in Patients with Schizophrenia. (2016). Archives of Psychiatric Nursing; 29(5):297-301. doi: 10.1016/j.apnu.2015.05.006. PMID: 26397432. 